\title{
Multimodal Imaging in a Case of Localized Suprachoroidal Hemorrhage
}

\author{
Avadhesh Oli, MD; Divya Balakrishnan, MD
}

Smt Kanuri Santhamma Center for Vitreo Retina Services, L. V. Prasad Eye Institute, Hyderabad, India

ORCID:

Avadhesh Oli: https://orcid.org/0000-0003-3421-3760

\section{Abstract}

Purpose: To report a case of localized suprachoroidal hemorrhage presenting as a choroidal mass.

Case Report: A 66-year-old woman presented with sudden onset pain in the right eye, one week following uneventful cataract surgery. The best corrected visual acuity (BCVA) was 20/160 and fundus examination showed a brown elevated choroidal mass temporal to the fovea in the right eye with normal retina and retinal vessels over it. The differential diagnoses considered were choroidal granuloma, melanoma, choroidal hemangioma, posterior scleritis, and localized suprachoroidal hemorrhage $(\mathrm{SCH})$. Fluorescein angiography (FA) and indocyanine green (ICG) angiography were unremarkable except for mild disc leakage; B-scan showed a choroidal mass with high surface reflectivity and low internal reflectivity, and optical coherence tomography (OCT) showed an elevation of retino-choroidal complex with hyporeflective mass in the outer choroid with choroidal folds suggestive of $\mathrm{SCH}$. Her systemic evaluation showed raised erythrocyte sedimentation rate (ESR) and consolidation in the upper lobe of the right lung. The patient did not take any additional treatment for her eye and the lesion regressed and visual acuity improved to $20 / 30$ in one month.

Conclusion: Delayed spontaneous suprachoroidal hemorrhage can present as a choroidal mass. Multimodal imaging helps to differentiate it from other sight-threatening and life-threatening ocular diseases.

Keywords: Choroidal Granuloma; Choroidal Mass; Melanoma; Supra Choroidal Hemorrhage

J Ophthalmic Vis Res 2020; 15 (1): 104-108

\section{Correspondence to:}

Avadhesh Oli, MD. Smt Kanuri Santhamma Center for Vitreo Retina Services L. V. Prasad Eye Institute, Hyderabad 500034, India.

E-mail: olieye@rediffmail.com

Received: 28-01-2019 Accepted: 28-07-2019

\section{Access this article online}

Website:

https://knepublishing.com/index.php/JOVR

DOI:

10.18502/jovr.v15i1.5956

\section{INTRODUCTION}

Spontaneous suprachoroidal hemorrhage (SCH) is a rare occurrence associated with intraocular

This is an open access journal, and articles are distributed under the terms of the Creative Commons Attribution-NonCommercial-ShareAlike 4.0 License, which allows others to remix, tweak, and build upon the work non-commercially, as long as appropriate credit is given and the new creations are licensed under the identical terms.

How to cite this article: Oli A, Balakrishnan D. Multimodal Imaging in a Case of Localized Suprachoroidal Hemorrhage. J Ophthalmic Vis Res 2020;15:104-108. 
surgeries. A few cases of $\mathrm{SCH}$ following Valsalva maneuver and delayed postoperative hemorrhage have been reported. ${ }^{[1]}$ A localized spontaneous $\mathrm{SCH}$ can cause diagnostic dilemma by presenting as a choroidal mass. Multimodal imaging including fluorescein angiography (FA), indocyanine green angiography (ICG), ultrasound B scan, and optical coherence tomography (OCT) help to differentiate various choroidal mass lesions like melanoma, choroidal granuloma, choroidal hemangioma, posterior scleritis, and suprachoroidal hemorrhage $(\mathrm{SCH})$. The present case highlights the role of multimodal imaging in differentiating $\mathrm{SCH}$ from other choroidal masses.

\section{CASE REPORT}

A 66-year-old woman presented with a history of sudden onset of excruciating pain in her right eye. She had undergone phacoemulsification and posterior chamber intraocular lens ( $\mathrm{PCIOL}$ ) implantation in the right eye a week before. Her systemic history was otherwise normal except for osteoarthritis for which she occasionally used analgesics. On examination, her visual acuity was 20/160 and 20/60 in right and left eyes, respectively. Slit lamp examination showed few Descemet's folds and PCIOL in the right eye and cataract in the left eye. Intraocular pressure (IOP) was 14 and $16 \mathrm{~mm} \mathrm{Hg}$ by applanation tonometry. Fundus showed a localized dome-shaped elevated choroidal mass temporal to the fovea with no orange pigment changes or subretinal fluid, with a diameter of about 5-6 disc diameters (DD), with overlying choroidal folds, and the presence of a few retinal hemorrhages in the temporal periphery in the right eye [Figure $1(A)]$. The retina of the left eye was normal. The patient was treated with topical steroids (prednisolone acetate eye drops $1 \%$ six times a day) and oral paracetamol 650 mg thrice a day. Keeping a differential diagnosis of melanoma, choroidal granuloma, choroidal hemangioma and posterior scleritis in mind, the patient was referred to the oncology clinic. The patient underwent multimodal imaging and systemic workup. FA showed normal fluorescence pattern, except for minimal disc leakage and overlying choroidal folds in the right eye [Figures 2 $(A-C)]$. ICG showed normal fluorescence pattern [Figures 2 (D-E)]. The fundus autofluorescence was normal in both eyes. Ultrasound B scan showed a well-defined mass with high surface reflectivity and low to medium internal reflectivity without sub-Tenon's fluid [Figure 1 (B)]. OCT showed an elevation of retinochoroidal complex over a hyporeflective space in the outer choroid with a smooth anterior surface, pushing the inner choroid, along with minimal subretinal fluid [Figures $1(C, D)]$. The absence of intrinsic circulation and a normal FA and ICG ruled out choroidal melanoma and hemangioma. Systemic workup showed a positive Mantoux test ( $24 \mathrm{~mm}$ ), and the chest X-ray showed lung consolidation in the upper lobe. The differential diagnoses considered were of either $\mathrm{SCH}$ or choroidal granuloma. She was referred to an internist to start antitubercular therapy (ATT). The patient came for a review at one month and had not taken any treatment for her eye other than topical steroids and paracetamol tablet. The lesion had spontaneously regressed and visual acuity had improved to $20 / 30$ with a normal OCT [Figure 2].

In retrospect, a choroidal mass with no changes on FA or ICG, which was dome shaped, optically lucent in OCT and B scan, showing high surface reflectivity with low internal reflectivity and a spontaneous resolution were suggestive of a localized $\mathrm{SCH}$. The typical FA and ICG features of choroidal granuloma ${ }^{[2]}$ (early hypo and late hyper-fluorescence in FA and persistent hypofluorescence in ICG) were not seen in this patient, but the disc showed minimal leakage in the late phase.

\section{DISCUSSION}

Spontaneous suprachoroidal hemorrhage is a relatively rare differential diagnosis of a choroidal mass lesion, which accounts for $2 \%$ of pseudo melanomas. ${ }^{[3]}$ The important risk factors are old age, hypertension, Valsalva maneuver and use of anti-coagulants. The dome-shaped elevation in the fundus due to $\mathrm{SCH}$ is usually limited to the area of fibrous adhesions concurrent with vortex veins; however, massive $\mathrm{SCH}$ can extend posteriorly. ${ }^{[4]}$ As it closely mimics other life-threatening conditions like choroidal melanoma, metastasis, and granuloma, patients are usually investigated. ${ }^{[2,3]}$

Though cataract surgery was uneventful in our patient, it could have been the precipitating factor. ${ }^{[5]}$ The patient not only presented with ocular 


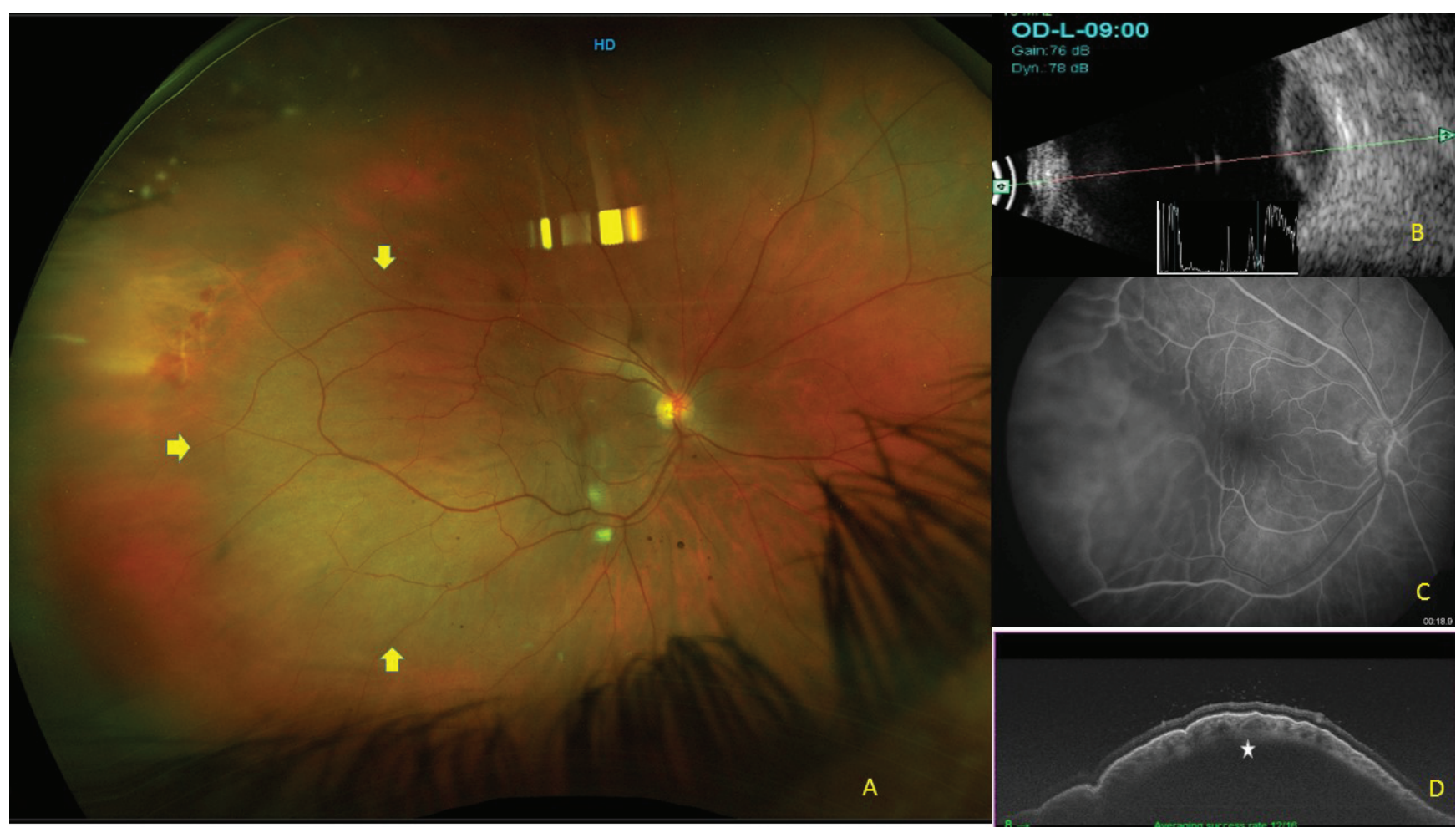

Figure 1. (A) Fundus picture: Yellow arrows show the extent of the lesion. (B) B scan shows a well-defined mass with high surface reflectivity and low internal reflectivity. No sub-Tenon's fluid. (C) FA and ICG show normal fluorescence and late disc leakage. (D) OCT shows elevation of the retino-choroidal complex with hyporeflective lesion in outer choroid with smooth anterior surface and a vertical scan showing choroidal folds.

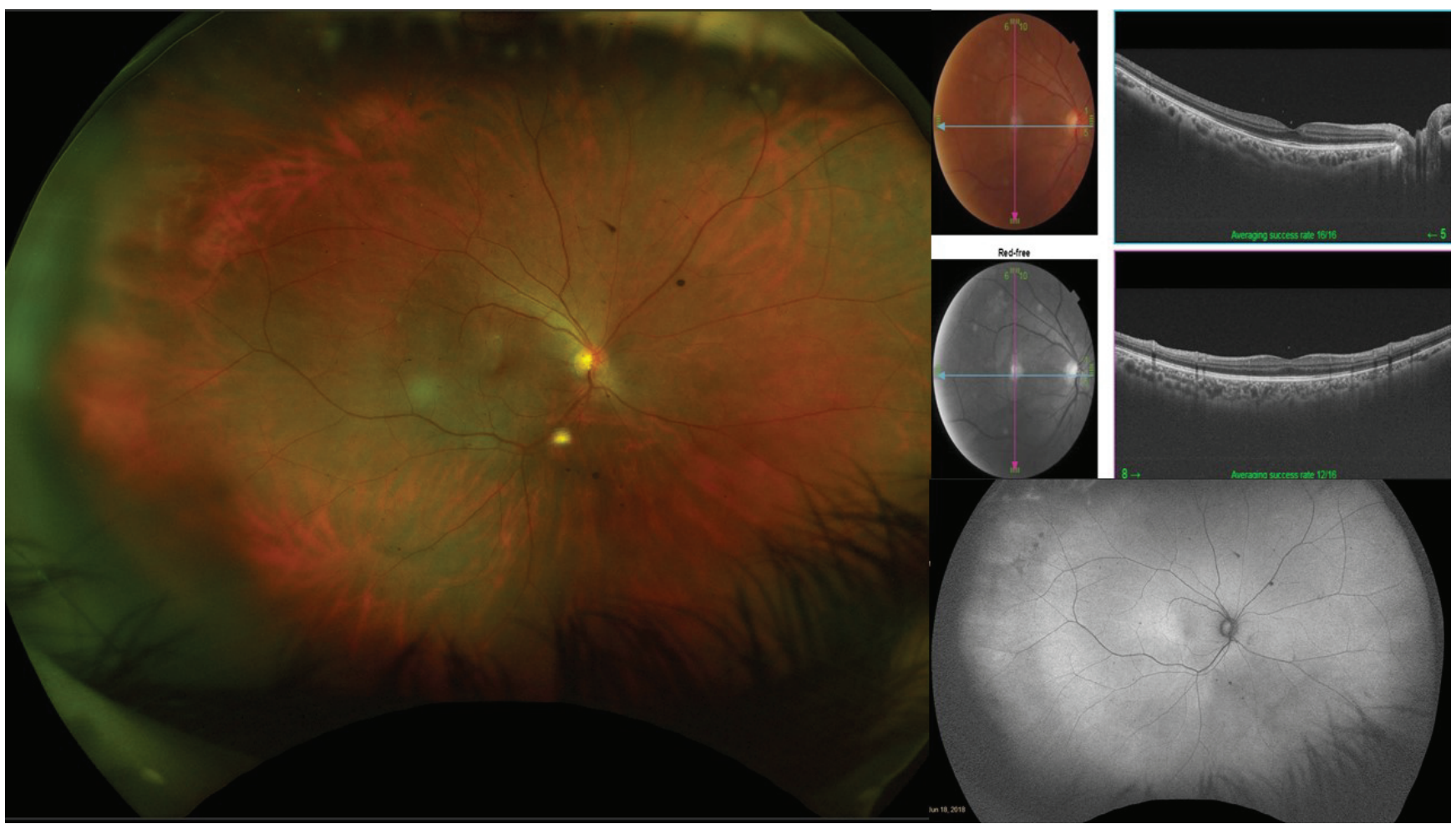

Figure 2. Fundus photo and OCT showing complete resolution of mass. 
Table 1. Key clinical and multimodal imaging features of differentials of choroidal mass

\begin{tabular}{|c|c|c|c|c|c|}
\hline Features & Melanoma & Granuloma & $\begin{array}{l}\text { Suprachoroidal } \\
\text { Hemorrhage }\end{array}$ & Hemangioma & Posterior scleritis \\
\hline \multicolumn{6}{|l|}{ Clinical features } \\
\hline Pain & No & Rare & Yes & No & Yes \\
\hline Choroidal folds & $\begin{array}{l}\text { May be seen at the } \\
\text { edges }\end{array}$ & Rare & Yes & No & Yes \\
\hline $\begin{array}{l}\text { B scan } \\
\text { Internal reflectivity }\end{array}$ & Low & Low & Echolucent & High & Low, T sign \\
\hline FFA ICG & Double circulation & $\begin{array}{l}\text { Early hypo } \\
\text { fluorescence, } \\
\text { late hyper } \\
\text { fluorescence } \\
\text { Persistent hypo } \\
\text { fluorescence }\end{array}$ & $\begin{array}{l}\text { No remarkable } \\
\text { features }\end{array}$ & $\begin{array}{l}\text { Early bright hyper } \\
\text { fluorescence, } \\
\text { late diffuse hyper } \\
\text { fluorescence } \\
\text { Early hyper } \\
\text { fluorescence, late } \\
\text { washout }\end{array}$ & $\begin{array}{l}\text { Pinpoint leaks } \\
\text { Zonal hyper with } \\
\text { pinpoint leak }\end{array}$ \\
\hline OCT & $\begin{array}{l}\text { Dome-shaped } \\
\text { mass, subretinal } \\
\text { fluid, Shaggy } \\
\text { photoreceptors }\end{array}$ & $\begin{array}{l}\text { Hypo reflective } \\
\text { thickening of } \\
\text { the choroid, the } \\
\text { outer boundary } \\
\text { of choroid can be } \\
\text { seen }\end{array}$ & $\begin{array}{l}\text { Hypo reflective } \\
\text { lesion in } \\
\text { outer choroid } \\
\text { with elevated } \\
\text { retinochoroidal } \\
\text { complex, } \\
\text { choroidal folds }\end{array}$ & $\begin{array}{l}\text { Elevated smooth } \\
\text { choroidal mass, } \\
\text { rounding of } \\
\text { choriocapillaris }\end{array}$ & $\begin{array}{l}\text { Increased } \\
\text { choroidal } \\
\text { thickness with } \\
\text { retinal folds }\end{array}$ \\
\hline
\end{tabular}

FFA, fundus fluorescein angiography; ICG, indocyanine green angiography; OCT, optical coherence tomography

pain, which is usually seen associated with posterior scleritis or granuloma, but also with features suggestive of $\mathrm{SCH}$, which caused a diagnostic dilemma. ${ }^{[1]}$ The fundus lesion of a brown elevated mass mimicked melanoma, hemangioma, and granuloma, but the features of melanoma-like orange pigments were absent.

Multimodal imaging helps to differentiate various choroidal mass lesions ${ }^{[6]}$ [Table 1].

The B scan showed acoustic hollowing, but no choroidal excavation. This feature of $\mathrm{SCH}$ has already been described..$^{[1,6]}$ The presence of choroidal folds over the lesion with background normal fluorescence in FA and choroidal folds over an optically empty elevated lesion in OCT is a feature of $\mathrm{SCH} .{ }^{[6,7]} \mathrm{A}$ choroidal hemangioma would show high surface and internal reflectivity in B scan and bright hyperfluorescence on FFA. The absence of typical features of granuloma in FA and OCT ruled out granuloma, but the disc leakage in the late phase of FA along with a history of pain created a diagnostic dilemma with granuloma and posterior scleritis. The disc leakage in this case, could be explained by postoperative inflammation.
A cautious interpretation of lab tests like Mantoux and meticulous analysis of multimodal imaging in correlation with clinical features is required to make the correct diagnosis of cases such as this one. Patients may be closely observed for one or two months for spontaneous resolution in case of strong clinical suspicion of localized $\mathrm{SCH}$, and invasive investigations and extensive systemic workup may be considered in cases of nonresolution. ${ }^{[6]}$

Even though localized $\mathrm{SCH}$ is a rare entity and regresses spontaneously, it is rarely thought of by ophthalmologists in the differential diagnosis of a choroidal mass. ${ }^{[7]}$

\section{Financial Support and Sponsorship}

Nil.

\section{Conflicts of Interest}

There are no conflicts of interest. 


\section{REFERENCES}

1. Marous $C L$, Sioufi K, Shields CL, Mashayekhi A, Shields JA. Coughing-induced suprachoroidal hemorrhage simulating melanoma in two cases. Retin Cases Brief Rep 2018;12:336-341.

2. Milea D, Fardeau C, Lumbroso L, Similowski T, Lehoang $\mathrm{P}$. Indocyanine green angiography in choroidal tuberculomas. Br J Ophthalmol 1999;83:753.

3. Shields JA, Mashayekhi A, Ra S, Shields CL. Pseudomelanomas of the posterior uveal tract: the 2006 Taylor R. Smith Lecture. Retina 2005;25:767-771.
4. Kahook MY, Noecker RJ. Why do choroidals form, and how do you treat them? Glaucoma Today 2007:3638.

5. Davison JA. Acute intraoperative suprachoroidal hemorrhage in capsular bag phacoemulsification. J Cataract Refract Surg 1993;19:534-537.

6. Augsburger JJ, Coats TD, Lauritzen K. Localized suprachoroidal hematomas. Ophthalmoscopic features, fluorescein angiography, and clinical course. Arch Ophthalmol 1990;108:968-972.

7. Skalka HW. Choroidal hemorrhage simulating malignant melanoma. J Clin Ultrasound 1982;10:190-192. 